\title{
Bovine lactoferrin reduces visceral fat and liver triglycerides in ICR mice
}

\author{
Satoru Morishita ${ }^{1,3 *}$, Tomoji Ono ${ }^{1}$, Chikako Fujisaki ${ }^{1}$, Yasuharu Ishihara ${ }^{1}$, \\ Michiaki Murakoshi, ${ }^{1,2}$, Hisanori Kato ${ }^{3}$, Masashi Hosokawa ${ }^{4}$, Kazuo Miyashita ${ }^{4}$, \\ Keikichi Sugiyama ${ }^{1,5}$ and Hoyoku Nishino ${ }^{2,5}$ \\ ${ }^{1}$ Research and Development Headquarters, Lion Corporation (100 Tajima, Odawara, Kanagawa 256-0811, JAPAN) \\ ${ }^{2}$ Kyoto Prefectural University of Medicine (Kawaramachi-Hirokoji, Kamigyou-ku, Kyoto 602-8566, JAPAN) \\ ${ }^{3}$ The University of Tokyo (1-1-1 Yayoi, Bunkyo-ku, Tokyo 113-8657, JAPAN) \\ ${ }^{4}$ Hokkaido University (3-1-1 Minatocho, Hakodate, Hokkaido 041-8611, JAPAN) \\ ${ }^{5}$ Ritsumeikan Global Innovation Research Organization, Ritsumeikan University (1-1-1 Nojihigashi, Kusatsu, Shiga 525-8577, JAPAN)
}

\begin{abstract}
Lactoferrin (LF) is a multi-functional glycoprotein found in milk. In a previous clinical trial, we showed that enteric-coated bovine LF (bLF) tablets could reduce visceral fat accumulation. We also showed that bLF had anti-adipogenic activity in vitro. However, the mechanisms responsible for these phenomena remain unclear. In this study, we established an animal model of visceral fat reduction via oral bLF administration. We used gastric intubation to ensure that LF was absorbed in the small intestine. bLF administration for 4 weeks significantly reduced mesenteric fat tissue $(P<0.05)$ and hepatic triglyceride levels $(P<0.01)$. Furthermore, these two outcomes were positively correlated $(R=0.581, P<0.05)$. Overall, these findings suggest that bLF affects mesenteric adipocytes and fatty acid metabolism in the liver.
\end{abstract}

Key words: lactoferrin, LRP1, mesenteric fat, visceral fat

\section{INTRODUCTION}

Metabolic syndrome is a combination of health disorders, such as high blood glucose, high blood pressure, and dyslipidaemia, caused by excess visceral fat accumulation, which ultimately progresses to cardiovascular disease and other chronic ailments. Recently, the number of individuals with metabolic syndrome has increased rapidly worldwide because of a shift towards dietary excess, lack of exercise, and increased stress, which has caused social problems. Visceral fat-type obesity is an underlying cause of metabolic syndrome.

Lactoferrin $(\mathrm{LF})$ is an iron-binding glycoprotein, which is present at high levels in breast milk. This protein has many known functions and is a potential antibacterial ${ }^{1)}$, antivi$\mathrm{ral}^{2)}$, immunostimulatory ${ }^{3)}$, antioxidant ${ }^{4)}$, and cancer preventive agent ${ }^{5}$. $\mathrm{LF}$ is a natural component of human breast milk, hence it is considered to be a safe food additive. Indeed, bovine $\mathrm{LF}$ (bLF) has been approved as a food additive in Japan while it is included in the "generally recognized as safe" category in the US. Recently, it was reported that plasma LF levels are inversely correlated with the severity of obesity as well as plasma triglyceride and free fatty acid(FA) levels in a clinical trial ${ }^{6)}$, suggesting that LF may ameliorate lipid metabolism dysfunctions. Several studies have already reported the beneficial effects of bLF on lipid metabolism in animals. Kawashima et al. reported that $2 \% \mathrm{LF}$ dietary supplementation significantly reduced serum non-esterified FA (NEFA) levels in 10-month-old C57BL/6Cr mice ${ }^{7)}$ while Takeuchi et al. showed that bLF improved many lipid metabolism-related parameters ${ }^{8)}$. After its administration to ICR mice, bLF reduced plasma triglyceride, NEFA, hepatic cholesterol, and hepatic triglyceride levels. However, these previous studies were not focused on visceral fat. Recently, two studies have demonstrated the in vitro effects of LF on preadipocytes, where LF suppressed the differentiation of adipocytes in MC3T3G2/PA6 cell lines ${ }^{9)}$ derived from the calvaria of mice ${ }^{10)}$, as well as the differentiation of 3T3-L1 adipocytes established from a mouse embryo ${ }^{11)}$. We reported the effects of bLF metabolites produced in the stomach and small intestine by the digestive enzymes pepsin and trypsin in an in vitro study $^{12)}$. In our previous study, full length and trypsin-digested LF inhibited the differentiation of preadipocytes derived from rat mesenteric fat, whereas pepsin-digested

\footnotetext{
*Correspondence to: Satoru Morishita, Research and Development Headquarters, Lion Corporation, 100 Tajima, Odawara, Kanagawa 256-0811, JAPAN

E-mail: smorishi@lion.co.jp

Accepted September 15, 2012 (recieved for review May 15, 2012)

Journal of Oleo Science ISSN 1345-8957 print / ISSN 1347-3352 online

http://www.jstage.jst.go.jp/browse/jos/ http://mc.manusriptcentral.com/jjocs
} 
LF did not. We also analyzed LF distribution using Sprague-Dawley rats, and orally administered bLF was found to be distributed mainly in the mesenteric fat tissue $^{12)}$. Based on these findings, we hypothesized that an enteric LF formulation would be effective for treating lipid metabolism dysfunctions, especially visceral fat reduction. Thus, we performed a double-blind clinical trial using enteric-coated bLF (eLF) tablets to avoid degradation in the stomach and found that bLF administration for 8 weeks reduced abdominal fat accumulation, especially visceral fat, in Japanese men and women with abdominal obesity ${ }^{13)}$.

However, the mechanisms responsible for visceral fat reduction remain unclear, and an appropriate animal model of visceral fat reduction is required to clarify the effects of eLF. Unfortunately, it was impossible to administer our eLF tablets to mice. Thus, we performed the present study where large doses of LF were administered by gastric intubation. A comparison of the routes of dietary intake and gastric intubation demonstrated that the latter is more efficient for ensuring that full-length LF reaches the intestine (data not shown). Thus, the objective of this study was to establish an animal model of visceral fat reduction by bLF administration by gastric intubation.

\section{EXPERIMENTAL PROCEDURES}

\subsection{Animals and diets}

All animal experiments were performed in accordance with the Lion Corporation (Japan) Guidelines for the Care and Use of Laboratory Animals. Seven-week-old male ICR mice were purchased from Japan SLC, Inc. (Shizuoka, Japan) and maintained in a temperature-controlled barrier room under a $12 \mathrm{~h}$ light/dark cycle, with free access to powdered laboratory chow (control diet; RD12450B, Research Diet Inc., New Brunswick, USA) and tap water. At 8 weeks of age, mice were divided into two groups with matched mean weights. One group was administered 0.5 $\mathrm{mL}$ distilled water (DW group) and the other was administered $100 \mathrm{mg}$ of bLF(Friesland Campina, Amersfoort, Netherlands) reconstituted in $0.5 \mathrm{~mL}$ distilled water ( $\mathrm{LF}$ group). Both were administered by gastric intubation at the same time, once each day. On day 28 , mice were fasted for $16 \mathrm{~h}$ and dissected under pentobarbital anesthesia. Blood samples were collected from the inferior vena cava to prepare serum. The mice were killed by exsanguination and mesenteric fat tissue and liver samples were collected and weighed.

\subsection{Analysis of lipids in serum and liver}

Serum total cholesterol, NEFA, triglyceride, and glucose levels were determined using Wako Cholesterol C-Test, NEFA C-Test, Triglyceride E-Test, and Glucose C-Test Kits (Wako Pure Chemical, Osaka, Japan), respectively. Lipids in the liver were extracted using the method of Folch et al. In brief, liver samples were dissolved in 2-propanol, and the hepatic triglyceride and total cholesterol levels were determined using the corresponding Wako Test kits.

\subsection{LF distribution}

Eight-week-old ICR mice were housed for 1 week and fasted for $16 \mathrm{~h}$. Subsequently, $100 \mathrm{mg}$ of bLF was administered to mice by gastric intubation. Mice were sacrificed under pentobarbital anesthesia and dissected at 15, 30, 60, and 120 min after bLF administration. Control mice were administered $0.5 \mathrm{~mL}$ distilled water by gastric intubation and sacrificed after $15 \mathrm{~min}$. Samples were prepared as described previously. bLF levels in the small intestine, mesenteric fat tissue, liver, and serum were quantified by ELISA and/or SDS-PAGE. LabWorks version 4.6.00.0 Image Acquisition and Analysis Software (Ultra-Violet Products Limited, Cambridge, UK) was used for quantification of fulllength LF in the gel images. The Bovine Lactoferrin ELISA Quantitation Kit(Bethyl Laboratory, Montgomery, TX, USA) and the ELISA Starter Accessory Package (Bethyl Laboratory) were used to determine immunoreactive LF (iLF) levels. SDS-PAGE was performed as described previously $^{12)}$.

\subsection{Statistical analysis}

Data were presented as means \pm standard deviations (SD). Most of the data from in vivo analyses were compared using unpaired Student $t$-tests. The ELISA iLF determinations were compared with the DW group using Dunnett's test. The level of statistical significance was set at $P<0.05$ and tests were conducted using JMP version 5.0.1a (SAS Institute Inc., Cary, NC, USA).

\section{RESULTS}

3.1 Effects of 4-week bLF administration on visceral fat accumulation and lipid metabolism-related parameters in ICR mice

Figures $1 \mathrm{a}$ and $1 \mathrm{~b}$ compare growth and body weight gains in the LF and DW groups over 4 weeks. The growth curves and weight gains in the LF group were slightly higher than those in the DW group. However, these differences were not statistically significant. Food intake was also not significantly different between the LF and DW groups (Fig. 1c). Serum NEFA, glucose, hepatic triglyceride, and total cholesterol levels were determined (Table 1). Mesenteric fat tissue weight and hepatic triglyceride levels in the LF group were significantly lower than those in the DW group (mesenteric fat tissue: $P<0.05$; hepatic triglyceride levels: $P<0.01)$. There were no significant differences among the other lipid metabolism-related parameters, but the weight of the mesenteric fat tissue was positively 


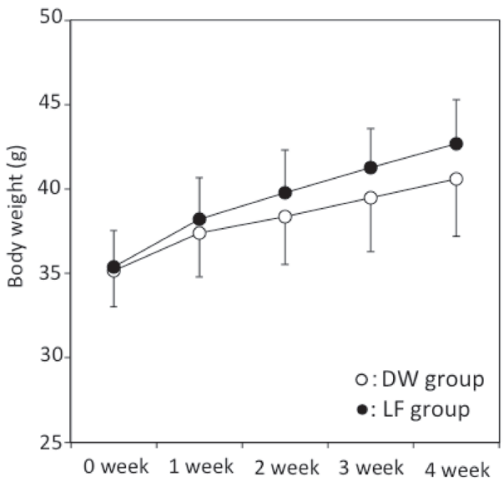

Fig. 1a

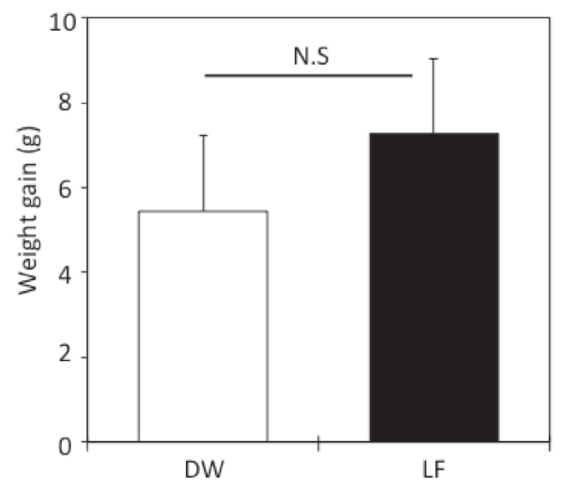

Fig. 1b

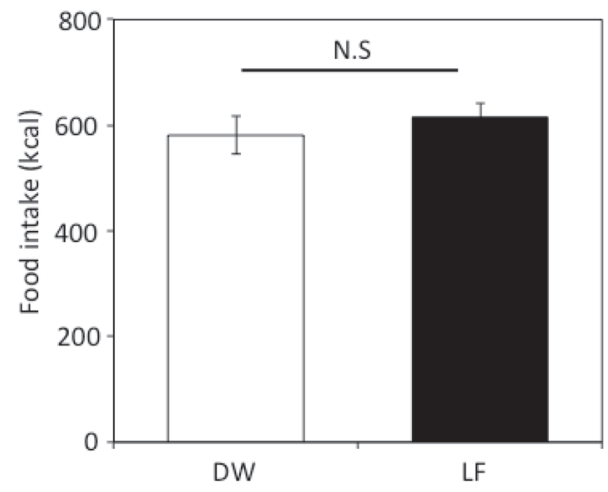

Fig. 1c

Fig. 1 Effect of orally administered bLF on body weight in ICR mice.

(a) Growth curves, (b) body weight gain over 4 weeks, (c) food intake over 4 weeks for LF and DW groups. The results are mean \pm SD (a), (b) and (c) $(n=9)$. DW group; $(\bigcirc)$ and LF group; $(\mathbf{O})$.

Table 1 Effects of bLF on the liver and mesenteric fat weight, hepatic lipid levels, and serum parameters.

\begin{tabular}{ccccc}
\hline & Measurement & DW & LF & $P$ \\
\hline \multirow{2}{*}{ Tissue weight } & Liver $(\mathrm{g})$ & $1.53 \pm 0.14$ & $1.67 \pm 0.24$ & 0.15 \\
& Mesenteric fat $(\mathrm{g})$ & $0.43 \pm 0.10$ & $0.28 \pm 0.12$ & $0.01^{*}$ \\
\hline \multirow{3}{*}{ Serum lipids } & NEFA $(\mathrm{mEq} / \mathrm{L})$ & $0.90 \pm 0.14$ & $0.83 \pm 0.21$ & 0.37 \\
& Glucose $(\mathrm{mg} / \mathrm{dL})$ & $111 \pm 19$ & $125 \pm 23$ & 0.18 \\
& Triglyceride $(\mathrm{mg} / \mathrm{dL})$ & $104 \pm 54$ & $91 \pm 51$ & 0.61 \\
& Cholesterol $(\mathrm{md} / \mathrm{dL})$ & $109 \pm 31$ & $104 \pm 23$ & 0.68 \\
\hline \multirow{2}{*}{ Liver lipids } & Triglyceride $(\mathrm{mg} / \mathrm{g}-$-liver $)$ & $16.3 \pm 5.5$ & $6.5 \pm 2.2$ & $0.0001^{* *}$ \\
& Cholesterol $(\mathrm{mg} / \mathrm{g}$-liver) & $1.4 \pm 0.7$ & $1.1 \pm 0.2$ & 0.14 \\
\hline
\end{tabular}

After oral administration of bLF for 4 weeks, mice were fasted for $16 \mathrm{~h}$ and dissected under pentobarbital anesthesia. Blood samples were collected from the inferior vena cava to prepare serum. The mice were killed by exsanguination and mesenteric fat and liver samples were collected and weighed. The serum lipid parameters were measured using Wako Test kits.

$* P<0.05, * * P<0.01$, compared with the DW group. Values are expressed as the mean $\pm \mathrm{SD}(\mathrm{n}=$ 9). Data were analyzed using unpaired t-tests with JMP software.

correlated with hepatic triglyceride levels $(\mathrm{R}=0.581$; Fig. 2).

\subsection{Distribution of orally administered bLF in the small intestine, mesenteric fat, liver, and blood}

Figure 3 shows the changes in LF levels in the small intestine after gastric intubation of $100 \mathrm{mg}$ LF to ICR mice. Pooled samples from the small intestine $(n=6)$ were analyzed by SDS-PAGE. Full-length bLF bands were observed at 15 and $30 \mathrm{~min}$ in the proximal halves of the small intestine but not in the distal halves (Figs. $3 a$ and 3b). After quantifying the full-length LF bands using a densitometer (Figs. 3c and 3d), we found that the maximum amount of full-length LF in the proximal halves of the small intestine was $0.38 \mathrm{mg}$ at $15 \mathrm{~min}$ after administration, which declined subsequently and the band was not detected at $60 \mathrm{~min}$.
Full-length LF was not detected in the distal halves of the small intestine at any time point. iLF was detected by ELISA in the proximal and distal halves of the small intestine at 15 and $30 \mathrm{~min}$ in the LF group but not in the DW group $(P<0.05$, Dunnett's test; Figs. $3 e$ and $3 f)$. The maximum iLF level was detected in the proximal half at 15 $\mathrm{min}$ and in the distal half at $30 \mathrm{~min}(1.33$ and $0.47 \mathrm{mg}$, respectively). iLF was detected in the mesenteric fat tissue at $15 \mathrm{~min}$ in the $\mathrm{LF}$ group $(P<0.05$, Dunnett's test vs DW group; Fig. 4). From 30 to $120 \mathrm{~min}$, the average iLF levels in the LF group were higher than those in the DW group, although these differences were not statistically significant. In the liver and serum, there were no differences in iLF levels at any time point compared with iLF levels in the DW group (data not shown). 


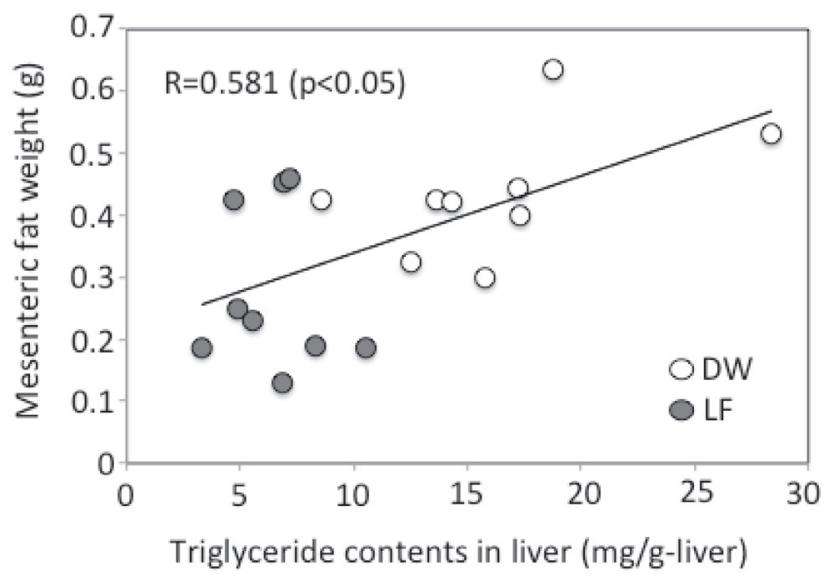

Fig. 2 Correlation between the weights of mesenteric fat tissues and hepatic triglyceride levels. DW group; $(\bigcirc)$ and LF group; $(\mathbf{)})$. Data were analyzed using a t-test with JMP software.

\section{DISCUSSION}

We previously reported the effects of eLF on visceral fat accumulation in a clinical trial ${ }^{13)}$. Thus, we attempted to establish an appropriate animal model for analyzing the mechanisms of visceral fat reduction. The results of the present study demonstrated that gastric intubation of bLF for 4 weeks significantly reduced accumulation of mesenteric fat tissue and hepatic triglyceride levels (Table 1). Several studies have already reported the beneficial effects of bLF on lipid metabolism in animals. Takeuchi et al. reported that $1 \% \mathrm{LF}$ supplementation in the normal diet significantly reduced plasma triglyceride, NEFA, hepatic triglyceride, and cholesterol levels in ICR mice ${ }^{8)}$. Kawashima et al. reported that $2 \%$ LF dietary supplementation significantly reduced serum NEFA levels in 10-month-old C57BL/6Cr mice ${ }^{7)}$. Unfortunately, these studies did not analyze visceral fat reduction. Shi et al. reported that bLF dietary supplementation significantly reduced the body fat ratios, including visceral fat, during 50 days of energy restriction ${ }^{14)}$. However, the condition of this experiment differed from our experimental design because food intake was not restricted. Therefore, the present study is the first to demonstrate visceral fat reduction due to bLF administration while fed an ad libitum diet.

In agreement with Takeuchi et al. ${ }^{8)}$, however, we observed no beneficial effects of bLF in a high fat diet-induced obese model (DIO model) using Sprague-Dawley rats where full-length bLF could reach the small intestine (data not shown). Their study also failed to demonstrate an effect of bLF on serum lipid parameters or hepatic triglyceride and total cholesterol levels. Further studies are necessary to confirm the mechanisms underlying these effects and to elucidate the influence of bLF in the DIO model.

We analyzed bLF distribution to confirm the presence of full-length LF in the small intestine in this model. In our previous study, $1000 \mathrm{mg}$ bLF reconstituted in distilled water was administered to Sprague-Dawley rats (mean weight $\sim 330 \mathrm{~g}$; data not shown). In the present study, the mean weight of ICR mice at 9 weeks after $16 \mathrm{~h}$ fasting was approximately $35 \mathrm{~g}$ (data not shown). The ratio of administered LF quantity per body weight was matched in the two experiments, hence $100 \mathrm{mg}$ bLF was administered. The maximum quantity of full-length bLF in the small intestine was $0.38 \mathrm{mg}(0.38 \%)$ (Fig. 3e). In this experiment, about $10 \%$ of full-length LF was detected at 15 min after administration while the ratio detected was less than one-tenth of that in the previous report. It is possible that the protease enzymatic activity in the stomach of mice was higher than that in rats, and that the residence time in the stomach of mice was longer than that of rats.

bLF was detected mainly in the mesenteric fat tissue (Fig. 4). Previously, we reported that bLF inhibited lipid accumulation in preadipocytes derived from rat mesenteric fat tissue as well as the upregulation of the genes for peroxisome proliferator-activated receptor gamma (PPAR $\gamma$ ) and CCAAT/enhancer-binding protein alpha, which are known to be regulators of differentiation of adipocytes ${ }^{12)}$. Yagi et al. and Moreno-Navarrete et al. reported similar effects of LF on the differentiation of 3T3L1 and MC3T3G2/PA6 cell lines into adipocytes ${ }^{9,11}$. This phenomenon may involve low-density lipoprotein-related protein 1 (LRP1). This protein is highly expressed in visceral fat and LRP1 silencing in 3T3F442A mouse preadipocytes significantly inhibits the expression of PPAR $\gamma^{15)}$. It is also known that LF is a binding protein of this receptor ${ }^{16)}$. LF binding to LRP1 might have a similar blocking effect to silencing adipocyte differentiation. In contrast, Grey et al. observed increased mitogenic signaling via LRP1 to p42/p44 MAPK (Erk) after LF stimulation in osteoblastic cells ${ }^{17}$. Indeed, it has been reported that long-lasting p42/p44MAPK signaling inhibits the differentiation of adipocytes ${ }^{18)}$.

In this study, visceral fat weight and hepatic triglyceride levels decreased significantly on bLF administration(Table. 1). And these outcomes were positively correlated (Fig. 2). LRP1 is also known to be a key receptor mediating the uptake of chylomicron remnants, which carry dietary lipids into the liver. It has been reported that bLF is transported into circulation from the intestine via the lymphatic pathway in rats ${ }^{19)}$ and orally administered bLF was detected in many mouse tissues, including the serum and liver ${ }^{20)}$. Crawford \& Borensztajn reported that intravenously administered bLF bound LRP1 in the liver and inhibited the plasma clearance of chylomicrons in mice ${ }^{211}$. It was also reported that endocytic absorption of chylomicron remnant lipids via LRP1 in adipose tissue was activated by insulin stimulation $^{22)}$. A crucial role for LRP1 in obesity was supported by a recent study, which showed that adipocyte $\mathrm{LRP}^{-1-}$ mice showed an overall decrease in fat mass and protection from $\mathrm{DIO}^{23)}$. Based on these results, it is possible 


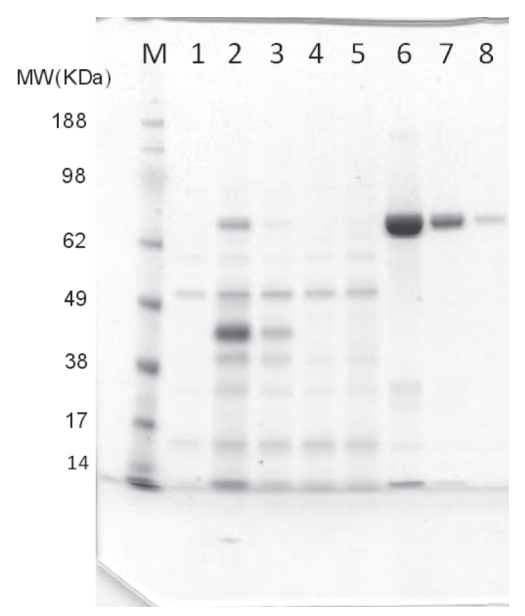

Fig. 3a

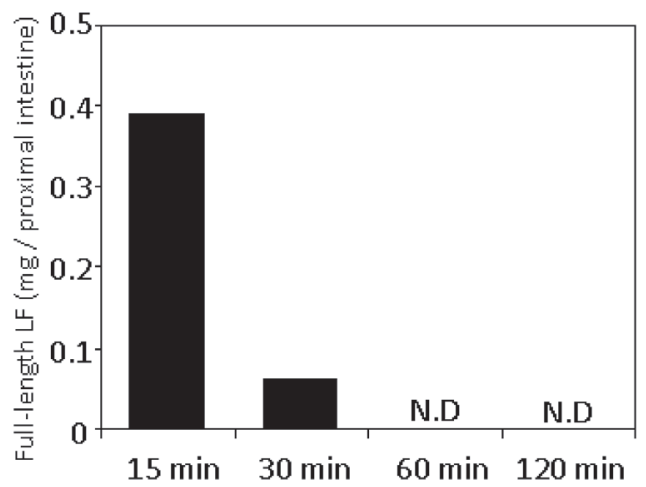

Fig. 3c

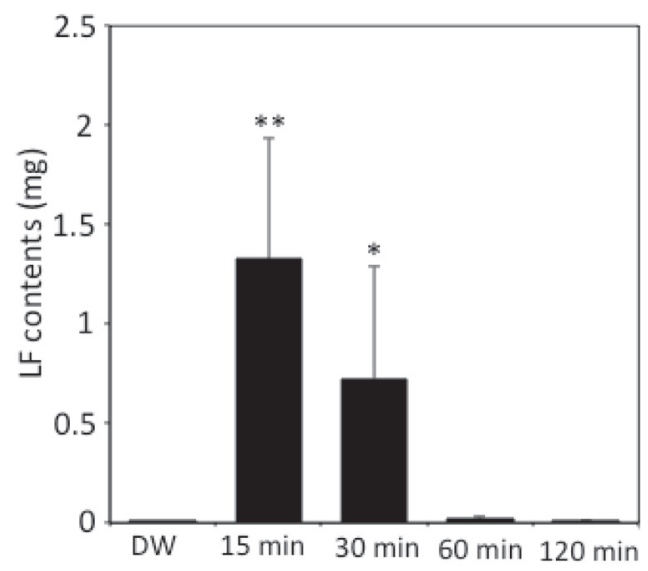

Fig. 3e

Fig. 3 bLF levels in the proximal and distal halves of the small intestine in the LF group.

Pooled samples $(n=6)$ were analyzed by SDS-PAGE. (a) SDS-PAGE image of bLF levels in the proximal half of the small intestine, (b) SDS-PAGE image of bLF levels in the distal half of the small intestine. M: molecular weight marker; lane 1: 15 min after gastric intubation with distilled water; lanes 2-5: 15, 30, 60, and 120 min after gastric intubation with LF; lanes 6-8: LF standard, 0.2, 0.05, and $0.01 \mathrm{mg} / \mathrm{mL}$. Full-length bLF in the (c) proximal and (d) distal halves of the small intestine was quantified using a densitometer. iLF levels (e) in the proximal and (f) distal halves of the small intestine were determined by ELISA. $* P<0.05$, $* * P<0.01$, compared with the DW group. The values are expressed as the mean \pm SD for six samples. Data were analyzed using Dunnett's test with JMP software.

Fig. $3 b$

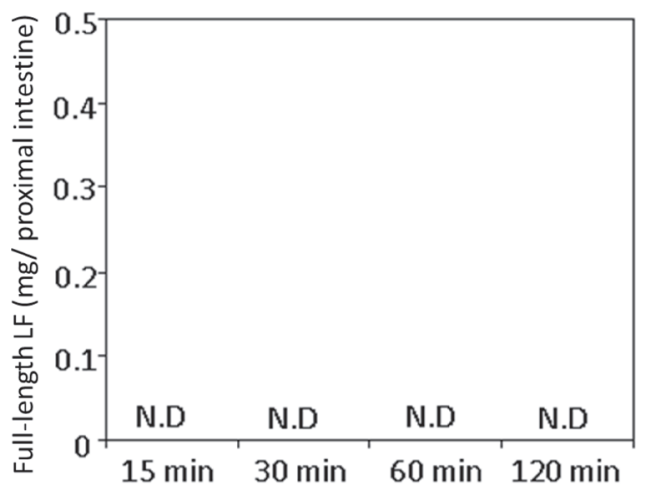

Fig. 3d

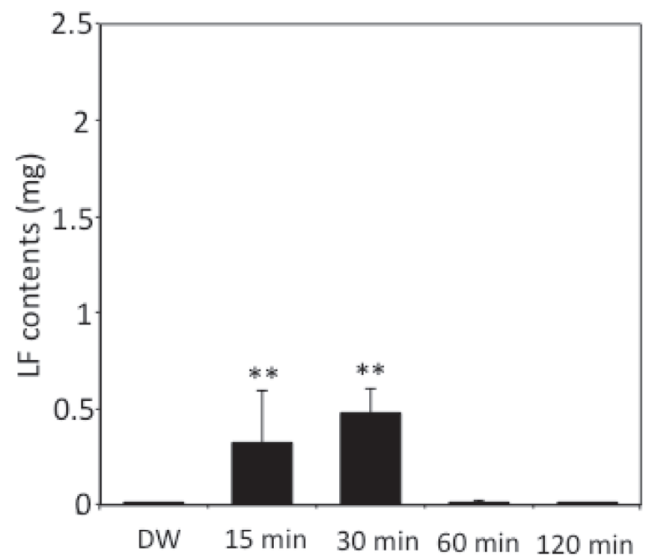

Fig. 3f

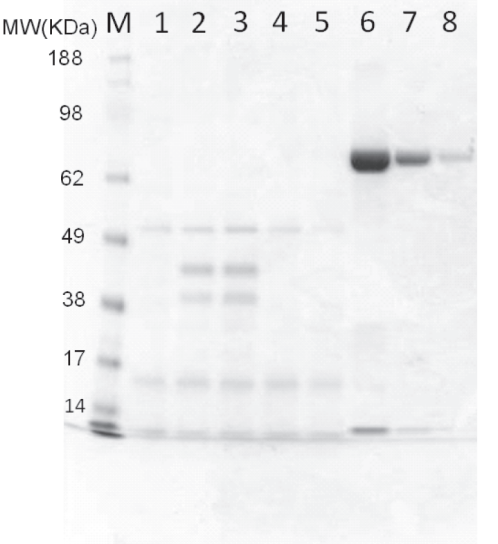




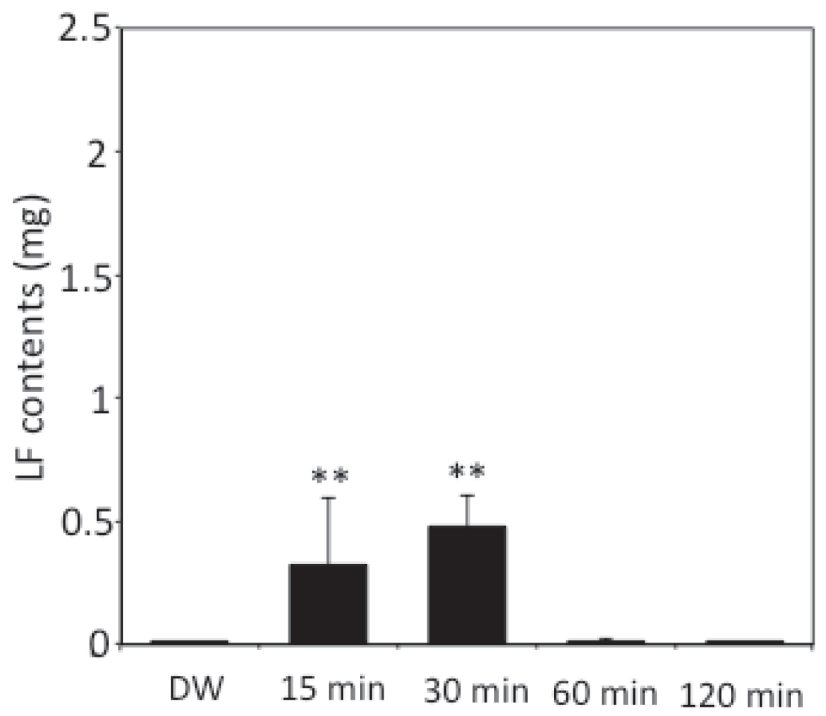

Fig. 4 bLF in mesenteric fat tissues in the LF group. Mesenteric fat tissues were homogenized in PBS containing $1 \%$ protease inhibitor cocktail. Samples were centrifuged and the supernatants were collected. iLF levels in mesenteric fat tissue were determined by ELISA. ${ }^{*} P<0.05$, compared with the DW group. Values are expressed as the mean \pm SD for six samples. Data were analyzed using Dunnett's test with JMP software.

that visceral fat and hepatic triglyceride reduction lead to binding of bLF to the LRP1 receptor, which inhibits the absorption of lipids via LRP1 in vivo. However, lipid-related parameters in the plasma were unaffected by bLF administration in our study. Therefore, it is very important to identify the tissues that have a key role in the metabolism of unclear lipids from the blood. Muscles, the main tissues of energy consumption, and the liver, the main tissue of fatty acid metabolism, should be analyzed to clarify the LF mechanism of action. It is also possible that LF has an effect on the small intestine. Takeuchi et al. reported reduced triglyceride levels in thoracic lymph after administration of a bLF-supplemented diet compared with normal diet, which suggests that bLF inhibits the absorption of dietary triglycerides ${ }^{8)}$. This mechanism is still unclear, but it is also a possible mechanism of bLF to reduce visceral fat. However, further studies are necessary to confirm bLF mechanisms underlying these observations.

\section{CONCLUSION}

We established an appropriate animal model to evaluate the beneficial effects of bLF on lipid metabolism. This is the first report to show visceral fat reduction after bLF ad- ministration in an animal model with unrestricted food intake. We demonstrated the utility of this model and clarified the mechanism whereby bLF reduces visceral fat and the risk of metabolic syndrome. Future studies should investigate the effects of bLF on gene and protein expression profiles especially in mesenteric fat tissues and the liver.

\section{ACKNOWLEDGMENTS}

This research received no specific grant from any funding agency in the public, commercial, or not-for-profit sector. The authors' contributions are as follows: S. M. and T. O. designed the study; M. M., H. K., M. H, K. M., K. S., and H. N. provided advice for the design and helped S. M. to write the manuscript; S. M., T. O., C. F., and Y. I performed the in vivo experiments and analyzed the data; and S. M. and T. O. investigated the distribution of LF and analyzed the data. All authors read and approved the final version of the manuscript. The authors have no conflicts of interest associated with the present study. The authors would like to thank Enago (www.enago.jp) for the English language review.

\section{References}

1) Tomita, M.; Bellamy, W.; Takase, M.; Yamauchi, K.; Wakabayashi, H. and Kawase, K. Potent antibacterial peptides generated by pepsin digestion of bovine lactoferrin. J Dairy Sci. 74, 4137-4142(1991).

2) Harmsen, M. C.; Swart, P. J.; de Bethune, M. P.; Pauwels, R.; De Clercq, E.; The, T. H. and Meijer, D. K. Antiviral effects of plasma and milk proteins: lactoferrin shows potent activity against both human immunodeficiency virus and human cytomegalovirus replication in vitro. J Infect Dis. 172, 380-388 (1995).

3) Zimecki, M.; Wlaszczyk, A.; Cheneau, P.; Brunel, A. S.; Mazurier, J.; Spik, G. and Kubler, A. Immunoregulatory effects of a nutritional preparation containing bovine lactoferrin taken orally by healthy individuals. Arch Immunol Ther Exp (Warsz). 46, 231-240 (1998).

4) Shoji, H.; Oguchi, S.; Shinohara, K.; Shimizu, T. and Yamashiro, Y. Effects of iron-unsaturated human lactoferrin on hydrogen peroxide-induced oxidative damage in intestinal epithelial cells. Pediatr Res. 61, 89-92 (2007).

5) Sekine, K.; Ushida, Y.; Kuhara, T.; Iigo, M.; Baba-Toriyama, H.; Moore, M. A.; Murakoshi, M.; Satomi, Y.; Nishino, H.; Kakizoe, T. and Tsuda, H. Inhibition of initiation and early stage development of aberrant crypt foci and enhanced natural killer activity in male rats administered bovine lactoferrin concomitantly with azoxymethane. Cancer Lett. 121, 211-216(1997). 
6) Moreno-Navarrete, J. M.; Ortega, F. J.; Bassols, J.; Castro, A.; Ricart, W. and Fernandez-Real, J. M. Association of circulating lactoferrin concentration and 2 nonsynonymous LTF gene polymorphisms with dyslipidemia in men depends on glucose-tolerance status. Clin Chem. 54, 301-309 (2008).

7) Kawashima, M.; Kawakita, T.; Inaba, T.; Okada, N.; Ito, M.; Shimmura, S.; Watanabe, M.; Shinmura, K. and Tsubota, K. Dietary lactoferrin alleviates age-related lacrimal gland dysfunction in mice. PLoS One. 7, e33148 (2012).

8) Takeuchi, T.; Shimizu, H.; Ando, K. and Harada, E. Bovine lactoferrin reduces plasma triacylglycerol and NEFA accompanied by decreased hepatic cholesterol and triacylglycerol contents in rodents. Br J Nutr. 91, 533-538(2004).

9) Yagi, M.; Suzuki, N.; Takayama, T.; Arisue, M.; Kodama, T.; Yoda, Y.; Numasaki, H.; Otsuka, K. and Ito, K. Lactoferrin suppress the adipogenic differentiation of MC3T3-G2/PA6 cells. J Oral Sci. 50, 419-425 (2008).

10) Kodama, H. A.; Amagai, Y.; Koyama, H. and Kasai, S. A new preadipose cell line derived from newborn mouse calvaria can promote the proliferation of pluripotent hemopoietic stem cells in vitro. $J$ Cell Physiol. 112, 89-95 (1982).

11) Moreno-Navarrete, J. M.; Ortega, F. J.; Ricart, W. and Fernandez-Real, J. M. Lactoferrin increases (172Thr) AMPK phosphorylation and insulin-induced (p473Ser) AKT while impairing adipocyte differentiation. Int $J$ Obes (Lond) . 33, 991-1000 (2009).

12) Ono, T.; Morishita, S.; Fujisaki, C.; Ohdera, M.; Murakoshi, M.; Iida, N.; Kato, H.; Miyashita, K.; Iigo, M.; Yoshida, T.; Sugiyama, K. and Nishino, H. Effects of pepsin and trypsin on the anti-adipogenic action of lactoferrin against pre-adipocytes derived from rat mesenteric fat. Br J Nutr. 105, 200-211 (2011).

13) Ono, T.; Murakoshi, M.; Suzuki, N.; Iida, N.; Ohdera, M.; Iigo, M.; Yoshida, T.; Sugiyama, K. and Nishino, H. Potent anti-obesity effect of enteric-coated lactoferrin: decrease in visceral fat accumulation in Japanese men and women with abdominal obesity after 8-week administration of enteric-coated lactoferrin tablets. $\mathrm{Br} \mathrm{J}$ Nutr. 104, 1688-1695 (2010).

14) Shi, J.; Finckenberg, P.; Martonen, E.; Ahlroos-Lehmus, A.; Pilvi, T. K.; Korpela, R. and Mervaala, E. M. Metabolic effects of lactoferrin during energy restric- tion and weight regain in diet-induced obese mice. Journal of Functional Foods. 4, 66-78(2012).

15) Masson, O.; Chavey, C.; Dray, C.; Meulle, A.; Daviaud, D.; Quilliot, D.; Muller, C.; Valet, P. and Liaudet-Coopman, E. LRP1 receptor controls adipogenesis and is up-regulated in human and mouse obese adipose tissue. PLoS One. 4, e7422 (2009).

16) Herz, J. and Strickland, D. K. LRP: a multifunctional scavenger and signaling receptor. J Clin Invest. 108, 779-784 (2001).

17) Grey, A.; Banovic, T.; Zhu, Q.; Watson, M.; Callon, K.; Palmano, K.; Ross, J.; Naot, D.; Reid, I. R. and Cornish, J. The low-density lipoprotein receptor-related protein 1 is a mitogenic receptor for lactoferrin in osteoblastic cells. Mol Endocrinol. 18, 2268-2278(2004).

18) Lii, C. K.; Huang, C. Y.; Chen, H. W.; Chow, M. Y.; Lin, Y. R.; Huang, C. S. and Tsai, C. W. Diallyl trisulfide suppresses the adipogenesis of 3T3-L1 preadipocytes through ERK activation. Food Chem Toxicol. 50, 478484(2012).

19) Takeuchi, T.; Kitagawa, H. and Harada, E. Evidence of lactoferrin transportation into blood circulation from intestine via lymphatic pathway in adult rats. Exp Physiol. 89, 263-270 (2004).

20) Fischer, R.; Debbabi, H.; Blais, A.; Dubarry, M.; Rautureau, M.; Boyaka, P. N. and Tome, D. Uptake of ingested bovine lactoferrin and its accumulation in adult mouse tissues. Int Immunopharmacol. 7, 1387-1393 (2007).

21) Crawford, S. E. and Borensztajn, J. Plasma clearance and liver uptake of chylomicron remnants generated by hepatic lipase lipolysis: evidence for a lactoferrinsensitive and apolipoprotein E-independent pathway. J Lipid Res. 40, 797-805 (1999).

22) Descamps, O.; Bilheimer, D. and Herz, J. Insulin stimulates receptor-mediated uptake of apoE-enriched lipoproteins and activated alpha 2-macroglobulin in adipocytes. J Biol Chem. 268, 974-981 (1993).

23) Hofmann, S. M.; Zhou, L.; Perez-Tilve, D.; Greer, T.; Grant, E.; Wancata, L.; Thomas, A.; Pfluger, P. T.; Basford, J. E.; Gilham, D.; Herz, J.; Tschop, M. H. and Hui, D. Y. Adipocyte LDL receptor-related protein-1 expression modulates postprandial lipid transport and glucose homeostasis in mice. J Clin Invest. 117, 3271$3282(2007)$. 